\title{
Comportamiento a fatiga de uniones a tope en chapas recubiertas soldadas por proceso láser
}

\author{
$\mathrm{J} . M^{\mathrm{a}}$. Amo ${ }^{(*)}$ y J. Durán ${ }^{(*)}$
}

\begin{abstract}
Resumen Se continúan los estudios de la resistencia a la fatiga y los mecanismos de rotura en uniones soldadas a tope por proceso láser, de chapas de acero con recubrimiento Al55-Zn, en espesores de 0,62 y 1,16 $\mathrm{mm}$. Las resistencias a fatiga a $2 \times 10^{6}$ ciclos para ambos espesores, se establecen finalmente en 130 y $175 \mathrm{MPa}$, respectivamente, y se confirma el gran tamaño de grano ferrítico - $300 \mu \mathrm{m}$ - producido por solidificación simétrica desde el centro de la soldadura. Se analizan las zonas de iniciación y propagación de las roturas y las microestructuras implicadas con MEB. Las primeras se corresponden con zonas de concentración de tensión elevada, fundamentalmente mordeduras o faltas de penetración, a partir de las cuales las grietas de fatiga progresan en semielipses, longitudinalmente y en sentido del espesor, por línea de fusión o ZAT. La rotura final se produce por desgarramiento del ligamento residual de la grieta de fatiga y desvío hacia la línea central de grano grueso de las soldaduras.
\end{abstract}

Palabras clave: Fatiga. Roturas. Láser. Soldaduras a tope.

\section{Fatigue behaviour of coated sheets butt joints welded by laser process}

\begin{abstract}
The fatigue strength and the fracture mechanism studies of butt laser welded joints in Al55-Zn coated steel sheets, 0.62 and $1.16 \mathrm{~mm}$ thick, have been continued. The $2 \times 10^{6}$ cycles fatigue strength for both thicknesses has been finally established in 130 and $175 \mathrm{MPa}$, respectively, and the big ferritic grain - $300 \mu \mathrm{m}$ - produced by symmetrical solidification from the fusion zone centre has been confirmed. Initiation and extension fracture zones are analysed by SEM and so are the involved microstructures. The first ones are concerned with high stress concentration zones, mainly undercuts or lack of penetration, from which the fatigue cracks extend in semiellipses, longitudinally and through thickness, along the fusion line or the HAZ. The final fracture is produced by tearing of the residual fatigue crack bound and crack deviation towards the central gross grain line of the welds.
\end{abstract}

Keywords: Fatigue. Fractures. Laser. Butt joints.

\section{INTRODUCCIÓN}

El amplio desarrollo conseguido en los últimos 15 años para los revestimientos anticorrosión en chapa de acero, desde los clásicos galvanizados a los recientes sistemas de protección basados en las combinaciones $\mathrm{Zn}-\mathrm{Ni}, \mathrm{Zn}-\mathrm{Fe}, \mathrm{Zn}-\mathrm{Al}$, en diferentes porcentajes, ha dado lugar a la aparición de nuevos productos como los electrogalvanizados (EZ), los galvanizados de alta temperatura (Galvaneal-Ga$4 / 10 \% \mathrm{Fe}$ ), y los de media y alta concentración de aluminio (Lavegal y Aluzink, Galvalume o Alga-

(*) Centro Nacional de Investigaciones Metalúrgicas, CENIM (CSIC), Avda. de Gregorio del Amo, 8. 28040-Madrid (España).
fort-AZ-30/55 \% Al). Numerosos estudios e investigaciones (1-3), han mejorado las propiedades de todos estos productos en muy variadas direcciones, tanto desde las perspectivas de anticorrosión y estéticas como mecánicas y de durabilidad. Estas propiedades, junto a otras no menos importantes, como conformabilidad, soldabilidad y resistencia a la fatiga, desarrolladas paralelamente, han favorecido su expansión en el mercado de estos materiales en tal grado que, hoy día, comprenden más de la mitad de la chapa fina utilizada en la industria.

Concordante con las propiedades antedichas, las industrias de mayor aplicación son las de la construcción, línea blanca, transporte y automovilística. Estas últimas, especialmente, exigen elevados grados de fiabilidad en la resistencia a la fatiga de los productos fabricados, en donde radica el gran 
interés de este tipo de estudios para las uniones soldadas. Aunque menos abundante, también existe variada información en este sentido, relativa, sobre todo, a la aplicación de procedimientos mejorados de soldadura por resistencia e incluso por proceso láser (4-8). Gran parte de los mismos están referidos, sin embargo, a diseños a solape, adecuados, en general, para la producción de aquellos tipos de industrias y para los espesores de más común utilización (0,4 a 1,5 mm).

Otra concepción diferente es la relativa a las uniones soldadas a tope, campo en el que la investigación ha sido más escasa y para las que solamente el proceso láser presenta posibilidades reales de aplicación, tanto por sus características de automatización, productividad, acceso por una sola cara, mínimo daño por calor, etc, (9-10), como por la posibilidad de conformación de los chapas soldadas (blanks). En este contexto, aprovechando la experiencia de investigaciones anteriores $(10$ y 11) y en línea con otras investigaciones en este sentido (12 y 13), se continúan en este trabajo los ensayos a fatiga en uniones láser a tope, en espesores de 0,65 y $1,16 \mathrm{~mm}$, y se estudian los mecanismos de fractura, iniciación y propagación, en relación con las microestructuras de las zonas soldadas.

\section{MATERIALES Y PROCEDIMIENTO EXPERIMENTAL}

El material de base utilizado ha sido chapa de acero extradulce, calidad para embutición profunda, recubierta por ambas caras con una capa de aproximadamente $35 \mu \mathrm{m}$ de aleación Al55-Zn producida por inmersión en caliente. Conocido comercialmente por diferentes nombres, Aluzink, Galvalume, etc., corresponde al producto de la nueva ACERALIA fabricado bajo el nombre de Algafort. La composición química de base y revestimiento y las propiedades mecánicas se dan en las tablas I y II, respectivamente.

Las soldaduras a tope se llevaron a cabo sobre muestras de $80 \times 150 \mathrm{~mm}$ (anchura $\times$ longitud), directamente sobre los bordes cortados a cizalla, con separación nula entre los mismos. Para evitar el

TABLA I.- Composición química del material (\% en masa $\times 1.000$ )

TABLE I.-Chemical composition of the material (wt. $\% \times 1,000)$

\begin{tabular}{|l|c|c|c|c|c|c|c|}
\hline & C & Mn & Si & P & S & Al & Zn \\
\hline $\begin{array}{l}\text { Base } \\
\text { Rev. }{ }^{(*)}\end{array}$ & 17 & 280 & $\begin{array}{c}<5 \\
1500\end{array}$ & 4 & 9 & $\begin{array}{c}<1 \\
5500\end{array}$ & $\begin{array}{c}- \\
43500\end{array}$ \\
\hline
\end{tabular}

(*) Valores nominales.
TABLA II.- Propiedades mecánicas

TABLE II.-Mechanical properties

\begin{tabular}{|c|c|c|c|}
\hline $\begin{array}{l}\text { Espesor } \\
(\mathrm{mm})\end{array}$ & $\begin{array}{l}\text { Lím. elástico } \\
(\mathrm{MPa})\end{array}$ & $\begin{array}{l}\text { Resistencia } \\
(\mathrm{MPa})\end{array}$ & $\begin{array}{l}\text { Elong. } \\
(\%)\end{array}$ \\
\hline 0,62 & 320 & 370 & 43 \\
1,16 & 350 & 400 & 39 \\
\hline
\end{tabular}

hundimiento de la zona fundida, se invirtieron las caras de los cortes en cada unión, con el fin de compensar el arrastre de material por la cizalla. Las muestras, limpias de polvo, grasas, etc., se sujetan en un posicionador móvil debajo del cabezal láser, provisto de protección por gas helio por las caras superior e inferior, que traslada las piezas a la velocidad de soldeo.

El equipo de soldeo es un Rofin-Sinar de $6 \mathrm{~kW}$ de potencia, que produce un láser continuo de $\mathrm{CO}_{2}$, equipado con una lente parabólica de $127 \mathrm{~mm}$ de longitud focal. Las soldaduras se realizaron en Modo TEM00, con mancha focal de 0,22 mm (spot de funcionamiento), localizada sobre la superficie de las piezas. Los parámetros de soldeo se dan en la tabla III.

Una vez soldadas, las uniones fueron exhaustivamente inspeccionadas visual, radiográficamente y por líquidos penetrantes, para estudiar la calidad de las soldaduras y localizar y definir los defectos existentes, mordeduras, faltas de fusión o penetración, porosidades, etc. En lo que concierne a esta presentación, los ensayos de fatiga se realizaron en una unidad MTS servohidráulica de $250 \mathrm{kN}$, estudiándose las superficies de las probetas fracturadas, bien directamente, en un MEB-Jeol modelo JXA840 - o bien sobre secciones o perfiles pulidos por microscopía óptica.

\section{ENSAYOS DE FATIGA}

Los ensayos de fatiga axial se han continuado sobre los mismos tipos de probetas ya utilizados en ensayos anteriores: sección reducida, $230 \mathrm{~mm}$ de

TABLA III.- Parámetros de soldeo de las soldaduras a tope

TABLE III.- Welding parameters of the butt welds

\begin{tabular}{|l|c|c|}
\hline \multirow{2}{*}{} & \multicolumn{2}{|c|}{ Espesores } \\
\cline { 2 - 3 } & $0,62 \mathrm{~mm}$ & $1,16 \mathrm{~mm}$ \\
\hline Potencia, $\mathrm{kW}$ & 0,8 & 1,3 \\
Velocidad, $\mathrm{m} / \mathrm{min}$ & 3,8 & 1,4 \\
Aporte térmico, J/mm & 12,6 & 55,7 \\
\hline
\end{tabular}

Nota: Gas plasma y protección superior: $\mathrm{He}, 14 \mathrm{~L} / \mathrm{min}$. Protección raíz: $\mathrm{He}, 4 \mathrm{~L} / \mathrm{min}$. 
longitud, con soldadura a tope en el centro orientada en dirección transversal al esfuerzo, de $24 \mathrm{~mm}$ de longitud. La ampliación del estudio se ha realizado básicamente en espesor de $0,62 \mathrm{~mm}$, dado que la problemática principal en trabajos a fatiga para este tipo de uniones se presenta para los espesores más delgados, a causa de la mayor sensibilidad a los defectos longitudinales, de tipo entalla, - mordeduras, faltas de penetración, etc. - muy comunes en soldaduras láser a tope.

Los resultados de los ensayos, realizados bajo carga cíclica repetitiva de razón $R=0,1$, se reproducen en la figura 1 , en la que se dan los números de ciclos para la rotura, $N$, en función de la tensión máxima aplicada durante el ensayo, en MPa. Se han representado también los resultados de los ensayos anteriores realizados en condiciones similares.

Las curvas definitivas de fatiga cumplen con las siguientes ecuaciones, estando la primera de ellas, para $0,62 \mathrm{~mm}$, corregida con estos últimos resultados:

Espesor de 0,62 mm ................... $\mathrm{NS}^{6}=1.12 \mathrm{E} 19$

Espesor de 1,16 mm ................ $\mathrm{NS}^{5}=4 \mathrm{E} 17$

La tensión máxima de trabajo a $2 \times 10^{6}$ ciclos, según la nueva curva para el espesor de 0,62 mm, resulta de unos $130 \mathrm{MPa}$, igual a la obtenida anteriormente. Para ambos espesores, por tanto, las tensiones máximas para aquella vida podrían establecerse, finalmente, en 130 y $175 \mathrm{MPa}$, respectivamente.

\section{MECANISMOS DE FRACTURA}

El inicio de la fatiga tiene lugar en zonas superficiales localizadas en el borde de la zona fundida (zona afectada térmicamente, ZAT) o por discontinuidades alargadas motivadas por defecto de desalineación del haz de láser o alteración local de parámetros de soldeo por formación de plasma (Figs. 2 y

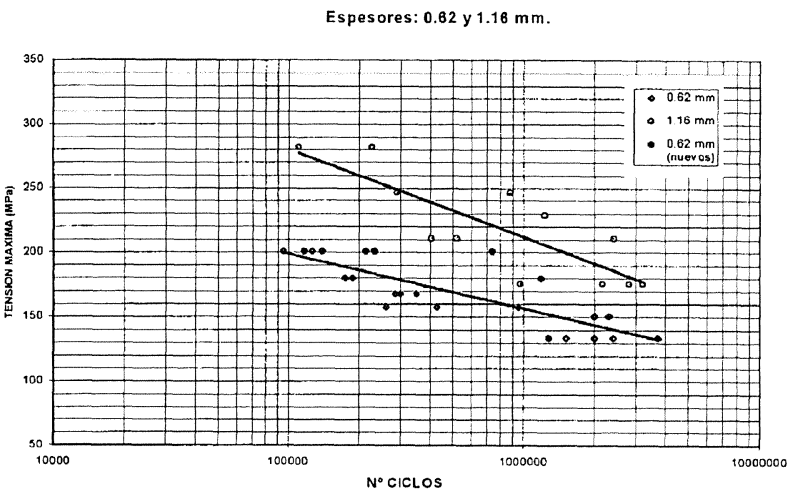

FIG. 1.- Curvas de fatiga de soldaduras a tope para los espesores de 0,62 y $1,16 \mathrm{~mm}$.

FIG. 1.- Fatigue results for 0.62 and $1.16 \mathrm{~mm}$ thick butt welds.

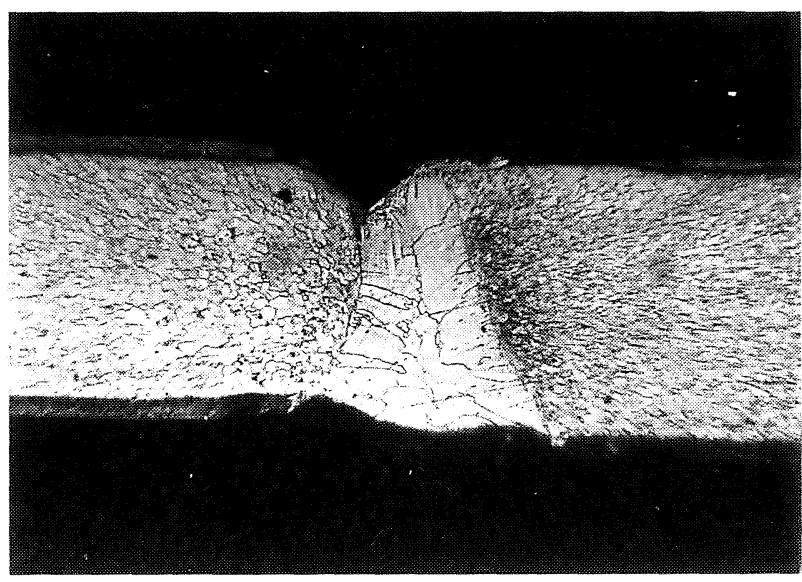

FIG. 2.- Estructuras metalográficas y defecto en borde de soldadura.

Fig. 2.- Metallographic structures and edge weld defect.

3), propagándose en sentido del espesor hasta alcanzar prácticamente su totalidad. La primera fotografía es, por otra parte, una excelente muestra del gran tamaño de grano ferrítico característico de las soldaduras en estos materiales. Las peculiares condiciones de enfriamiento del ciclo láser de soldeo originan una solidificación simétrica desde el centro del metal fundido. El tamaño de grano de esta zona, de 300 a $500 \mu \mathrm{m}$, contrasta con el grano afinado de la ZAT unas $20 \mu \mathrm{m}$. Las entallas se producen en estas zonas o en líneas de fusión, a través de las cuales se origina el crecimiento inicial de fatiga.

En el crecimiento longitudinal posterior de estas grietas por el borde de soldadura, se originan cambios ocasionales de dirección en forma de escalones hacia la zona central del metal fundido (Fig. 4), favorecidos por el grueso tamaño de grano de dicha zona. Incrementos locales de tensión facilitan la

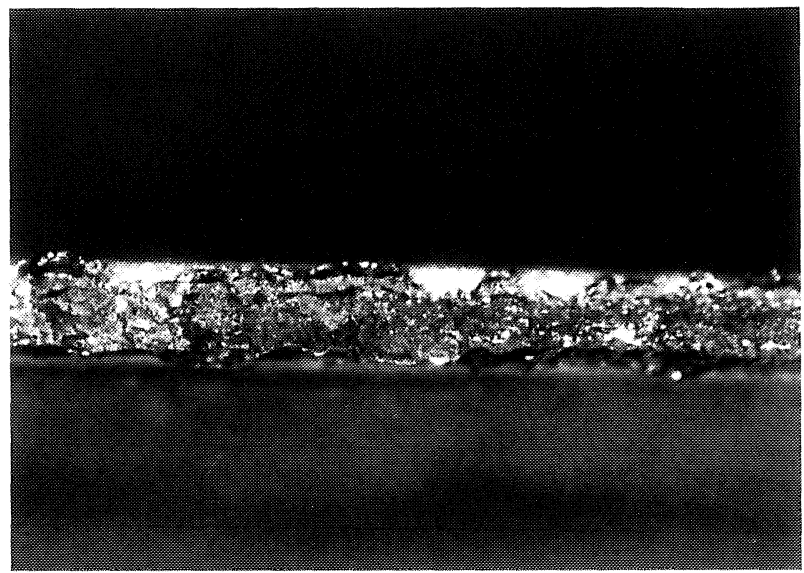

FIG. 3.- Lunetas que muestran el desarrollo inicial de las grietas de fatiga.

FIG. 3.- Lenticular zones showing the initial development of fatigue cracks. 


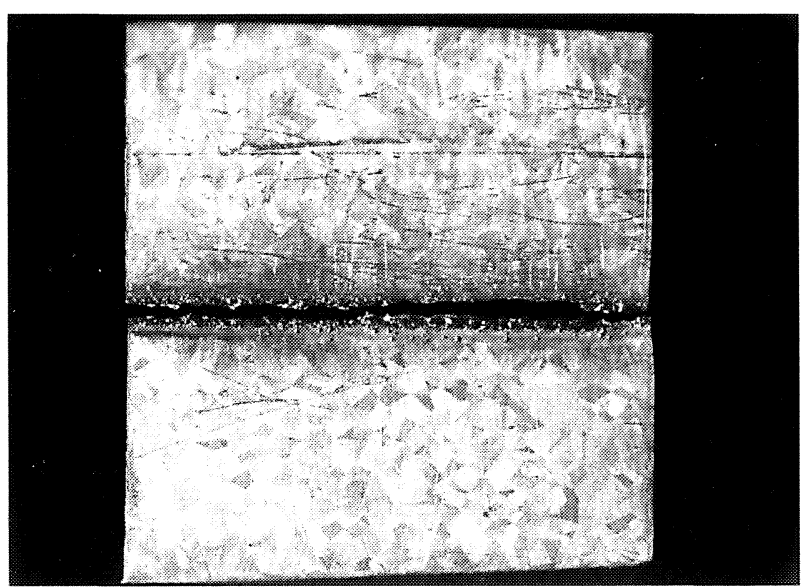

FIG. 4.- Trayectoria completa de la rotura

\section{FIG. 4.-Complete path of the rupture.}

propagación de la grieta por la zona fundida, preferentemente por la zona central más débil de confluencia de los granos.

En las roturas, se observan claramente los puntos de iniciación de las fracturas (Fig. 5a), que pueden alcanzar profundidades de hasta $70 \mu \mathrm{m}$. Se observa, asimismo, en esta fotografía, la propagación de la fatiga a través del espesor, hasta la zona en cúpulas por coalescencia de microvacíos del ligamento residual -zona clara de la izquierda de la foto- que se produce en la rotura final rápida por desgarre. La desviación hacia el centro del metal fundido, mediante escalones de rotura dúctil y descohesiones del grano grueso, se ve en la figura $5 b$. La rotura final rápida por esta zona es transcristalina, de tipo frágil (Fig. 6a). Hacia los extremos de las probetas, se produce la rotura definitiva por desgarre del material (zona en cúpulas de la figura $6 b$ ).

A veces, también la fatiga se propaga a través de las zonas de grano grueso próximas a la línea de fusión o ZAT. En la figura 7a, se aprecia el avance de la fatiga por uno de estos granos, cuya estriación se amplía en la figura $7 \mathrm{~b}$.

\section{CONCLUSIONES}

La soldadura láser a tope de chapas finas recubiertas de aleación A155-Zn da lugar a un elevado tamaño de grano en la zona fundida, con solidificación simétrica respecto al plano central de la sección de soldadura.

El desarrollo del proceso de fatiga está muy influido por la existencia de defectos superficiales en : . borde de fusión. Los resultados obtenidos para la vida de fatiga de este tipo de uniones a $2 \times 10^{6}$ ciclos se consideran muy aceptables (130 MPa para espesor de 0,62 $\mathrm{mm}$ y $175 \mathrm{MPa}$ para $1,16 \mathrm{~mm}$ ).

$\mathrm{La}$ iniciación de las roturas tiene lugar por la ZAT, propagándose por ésta hasta cambiar de direc-
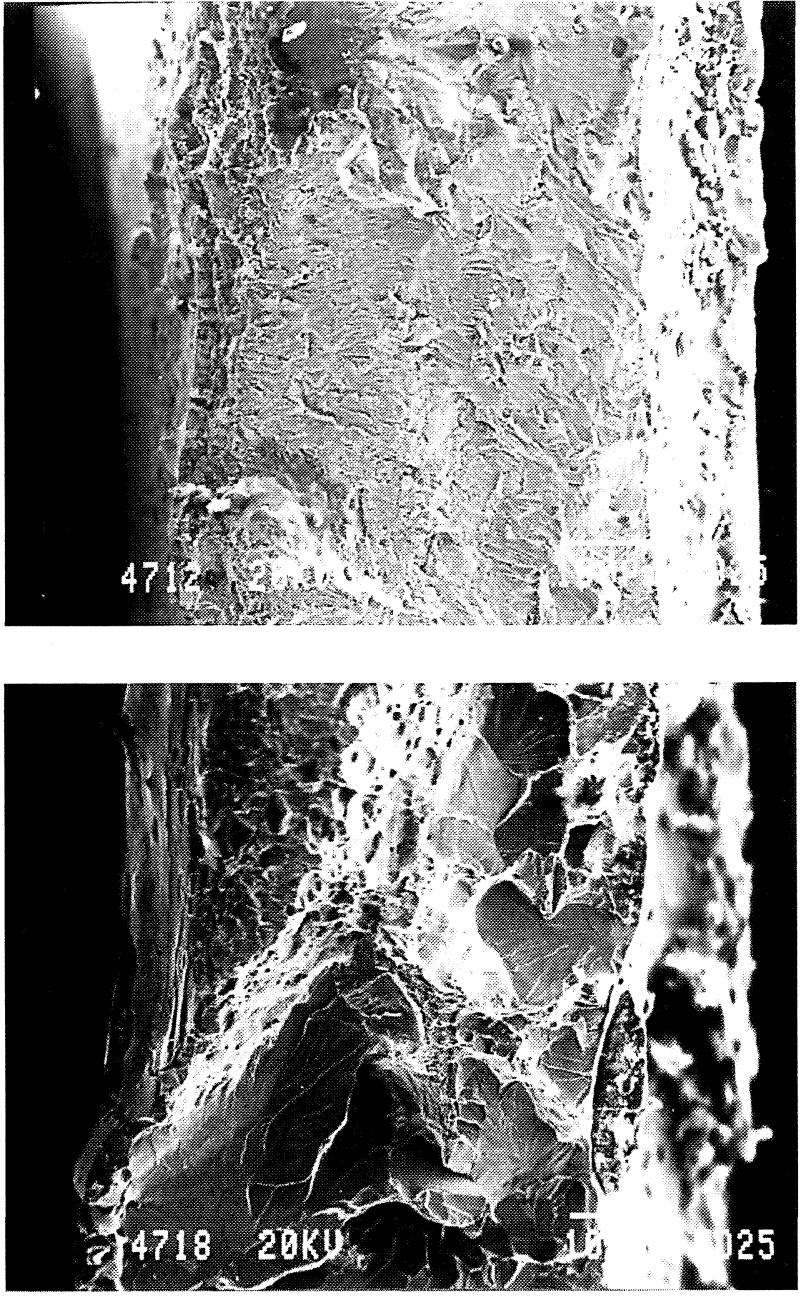

FIG. 5.- a) Zonas de iniciación, propagación y rotura final en cúpulas. $b$ ) Escalón de desvío a la zona central fundida.

FIG. 5.- a) Initial zones, propagation and dimple final rupture. b) Deviation step to the central melted zone.

ción hacia la zona fundida de grano grueso, a causa del aumento de la concentración local de las tensiones. Por esta zona, la grieta continúa en propagación transcristalina, completándose las roturas hacia los extremos de las probetas en forma dúctil, por coalescencia de microvacíos.

\section{REFERENCIAS}

(1) Townsend, H.E. BIEC CLIPS, 32, Sep. 1987: 37-41.

(2) Bergström, U. y Engberg, G. 2nd Intern. Conf. on Zinc Coated Steel Sheet. Zinc Development Assoc. (Londres). Roma, 9-10 Jun. 1988, SC5/1.

(3) Massinon, D. ECSC Research Project, 7210-KB/309, 1991.

(4) Natal, T. e Irving, B. Weld. J, Mar., 1992: 47-52.

(5) Defourny, J., Monfort, G. y Bragard, A. Intern. Inst. of Welding. Doc. IIW-III-894-87, 1987.

(6) Flavenot, J.F., Deville, J.P., Diboine, A., Cantello, M. у Goввi, S.L. Weld. World. 31 (5), 1993: 358-361. 

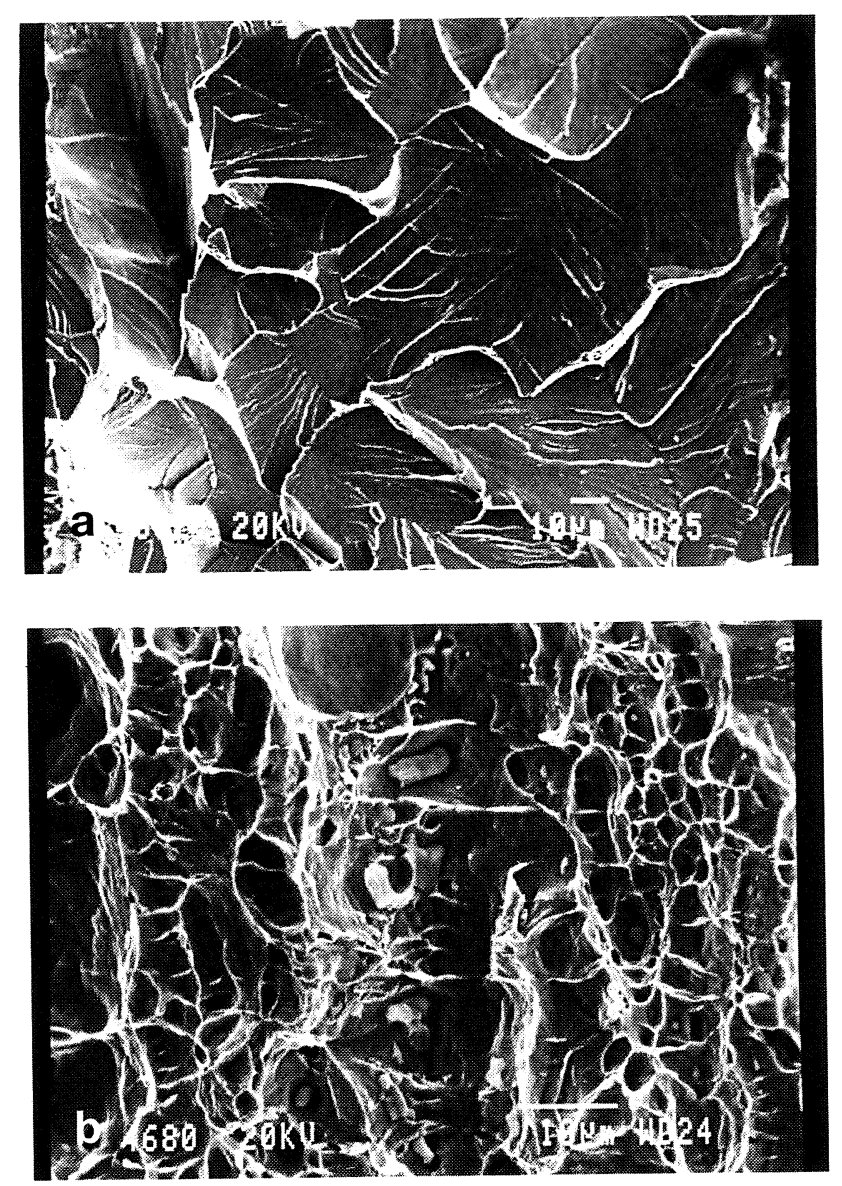

FIG. 6. - a) Zona de rotura en material fundido. b) Zona de desgarro final dúctil.

FIG. 6. - a) Rupture zone in melted material. b) Final ductile tearing zone.

(7) Wang, P.C. y EwING, K.M.. Weld. J., Oct., 1991: 43-47.

(8) Rustia, V. ECSC Research Project 7210-Ka/813, 1993.

(9) Bhatnagar, R.S., Bingaman, M.J. y McShane, M.D. Microstruc. Sci. 20, 1993: 65-85.

(10) Amo, J.M., Duran, J., Chao, J. y Fernandez-Saez, J. J. Mater. Sci., 31, 1996: 6.595-6.607.

(11) Amo, J.M., Duran, J. y Chao, J. Anal. Mecánica Fractura, 12, 1995: 178-184.
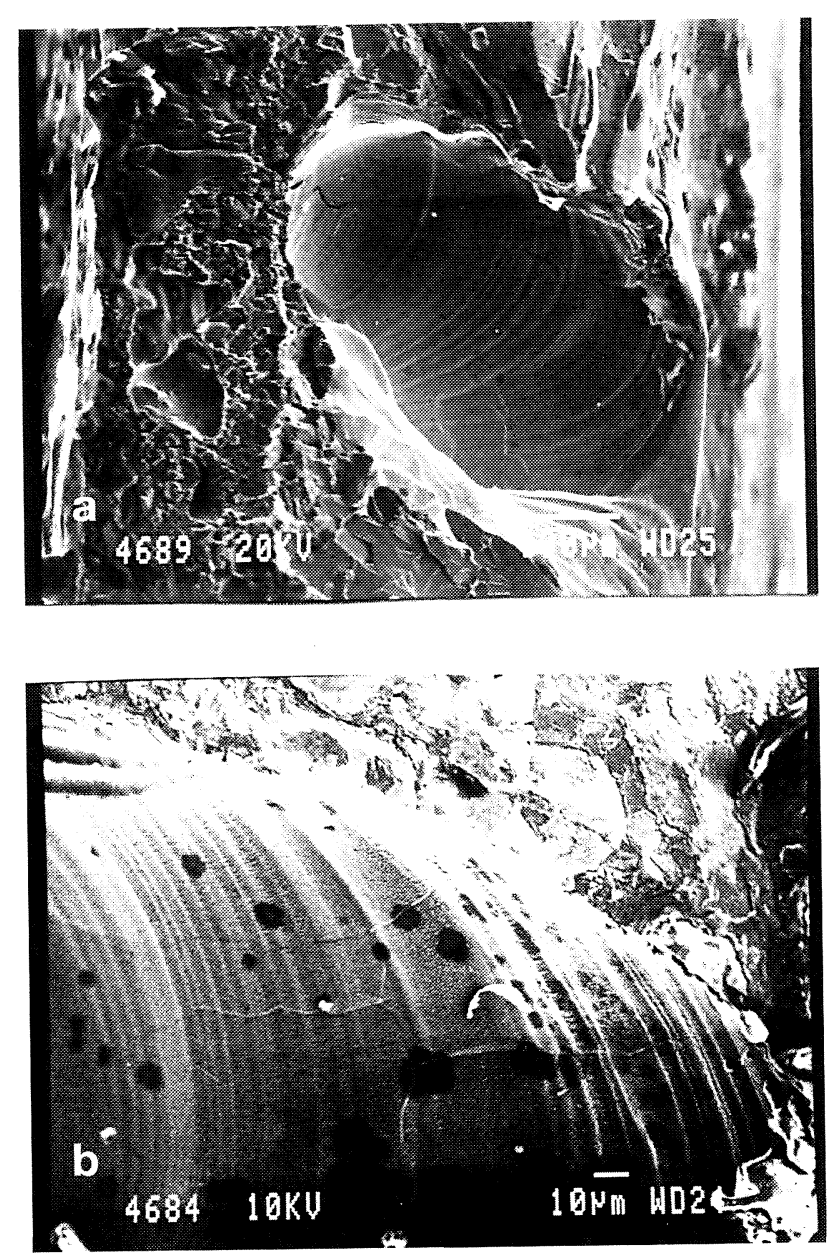

FIG. 7.- a) Grano grueso cortado por fatiga. b) Ampliación del grano anterior.

FIG. 7.- a) Fatigue cut gross grain. b) Enlargement of the former grain.

(12) Andrews, R.M. y Punchou, C.S. Welding Institute Report, 408, 1989.

(13) Miki, C., Sakano, M., Toyoda, Y y Yoshizawa, T. IIWXIII-1384-90, 1990. 